\title{
Combined High Resistin and EGFR Expression Predicts a Poor Prognosis in Breast Cancer
}

\author{
Yue Zeng, ${ }^{1,2}$ Chih-Hsin Tang, ${ }^{3,4,5}$ Yan Wang, ${ }^{6}$ Hua-Jun Lu, ${ }^{7}$ Bi-Fei Huang, ${ }^{2}$ Qian Wang, ${ }^{2}$ \\ Jun-Kang Shao, ${ }^{2}$ Lu-Lu Jin, ${ }^{8}$ Chao-Qun Wang $\mathbb{D}^{2},{ }^{2}$ and Li-Song Teng $\mathbb{D}^{1}$ \\ ${ }^{1}$ Department of Surgical Oncology, The First Affiliated Hospital, Medical School of Zhejiang University, Hangzhou, Zhejiang, China \\ ${ }^{2}$ Department of Pathology, Affiliated Dongyang Hospital of Wenzhou Medical University, Dongyang, Zhejiang, China \\ ${ }^{3}$ Graduate Institute of Basic Medical Science, China Medical University, Taichung, Taiwan \\ ${ }^{4}$ Department of Pharmacology, School of Medicine, China Medical University, Taichung, Taiwan \\ ${ }^{5}$ Department of Biotechnology, College of Health Science, Asia University, Taichung, Taiwan \\ ${ }^{6}$ Department of Medical Oncology, Affiliated Dongyang Hospital of Wenzhou Medical University, Dongyang, Zhejiang, China \\ ${ }^{7}$ Department of Oncological Radiotherapy, Affiliated Dongyang Hospital of Wenzhou Medical University, Dongyang, \\ Zhejiang, China \\ ${ }^{8}$ Laboratory of Biomedicine, Affiliated Dongyang Hospital of Wenzhou Medical University, Dongyang, Zhejiang, China
}

Correspondence should be addressed to Chao-Qun Wang; chaoqunw869@163.com and Li-Song Teng; 1steng@zju.edu.cn

Yue Zeng and Chih-Hsin Tang contributed equally to this work.

Received 9 September 2020; Revised 27 October 2020; Accepted 18 November 2020; Published 29 November 2020

Academic Editor: Kyoung-Ho Pyo

Copyright $\odot 2020$ Yue Zeng et al. This is an open access article distributed under the Creative Commons Attribution License, which permits unrestricted use, distribution, and reproduction in any medium, provided the original work is properly cited.

\begin{abstract}
Elevated levels of resistin and epidermal growth factor receptor (EGFR) facilitate the development of breast cancer, although there are no reports of any correlation between these proteins. This study analyzed 392 human breast cancer tissue specimens and 42 samples of adjacent normal tissue. Rates of positive and strongly positive resistin expression were significantly higher in breast cancer tissue than in the adjacent nontumor tissue $(83.2 \%$ vs. $23.8 \%$ and $20.9 \%$ vs. $0.0 \%$, respectively; $P<0.001$ for both comparisons). Positive resistin expression was significantly associated with tumor size, grade, stage, estrogen receptor (ER), progesterone receptor (PR), human epidermal growth factor receptor 2 (HER2) status, and molecular classification; strongly positive resistin expression was associated with tumor grade, ER, PR, HER2 status, and molecular classification. Significantly positive correlations were observed between positive and strongly positive resistin expression and corresponding levels of EGFR expression. Relapse-free and overall survival was worse for patients with high levels of both proteins than for those with high levels of only one protein or normal levels of both proteins. Our evidence suggests that combined high levels of resistin and EGFR expression correlate with survival in patients with breast cancer.
\end{abstract}

\section{Introduction}

Elevated levels of circulating resistin have been linked to a higher risk of breast cancer $[1,2]$, and significantly elevated serum resistin has been documented in patients with breast cancer [3-6]. Significant correlations have been observed between high resistin expression in breast cancer tissue and tumor stage, tumor size, lymph node metastasis, estrogen receptor (ER) status, and poor survival [7]. We have previously reported finding much higher levels of resistin expression in breast cancer tissue than in normal breast tissue [8], but in that study, we did not analyze the relationship between the high expression of resistin and the clinicopathological characteristics of breast cancer patients.

Epidermal growth factor receptor (EGFR), a member of the HER family of receptor tyrosine kinases, is abnormally 
expressed and activated in many epithelial tumors and plays a critical role in the initiation and development of cancer via modulating downstream signaling pathways $[9,10]$. Our previous investigations echo other reports describing how resistin and EGFR promote tumor progression through downstream p44/p42 MAPK (ERK1/2) signaling [11, 12]. Resistin can also increase EGFR phosphorylation through the Toll-like receptor 4 (TLR4)/Src pathway and thereby promote lung adenocarcinoma metastasis [13]. However, no reports exist as to any association between resistin and EGFR in breast cancer.

This paper describes our insights derived from immunohistochemistry (IHC) analyses of resistin and EGFR expression in breast cancer and healthy normal breast tissue specimens obtained from 392 Chinese Han women. We detail the clinical prognostic significance of a positive association between resistin and EGFR expression in breast cancer.

\section{Materials and Methods}

2.1. Patients and Tissue Samples. Breast cancer tissue samples were obtained from 392 untreated Chinese Han women aged 24-90 years (median 50 years) who underwent surgery in the Affiliated Dongyang Hospital of Wenzhou Medical University (Dongyang, Zhejiang, China) between 2007and 2019. Forty-two samples of adjacent normal breast tissue were also obtained following surgical resection. Pathohistological diagnoses followed the World Health Organization breast tumor classification criteria [14]. Histological grading was based on the Scarff-Bloom-Richardson system [15]. According to ER, progesterone receptor (PR), human epidermal growth factor receptor 2 (HER2), and Ki-67 status, the samples were classified into 4 molecular subtypes [11, 16, 17]: luminal A $\left(\mathrm{ER}^{+} / \mathrm{PR}^{+}[\geq 20 \%] / \mathrm{HER}^{-}, \mathrm{Ki}-67<14 \%\right)$; luminal B, containing hormone receptor-positive cases that did not meet the conditions of luminal A; HER2-enriched ( $\mathrm{ER}^{-}, \mathrm{PR}^{-}$, and $\left.\mathrm{HER} 2^{+}\right)$; or triple-negative breast cancer (TNBC) $\left(\mathrm{ER}^{-}\right.$, $\mathrm{PR}^{-}$, and $\mathrm{HER} 2^{-}$). Follow-up information was available for 239 breast cancer patients with a median follow-up time of 60 months (range, 6-72 months). The Ethics Committee of the Affiliated Dongyang Hospital of Wenzhou Medical University approved this study. All study methods satisfied the relevant guidelines and regulations issued by the Affiliated Dongyang Hospital of Wenzhou Medical University.

2.2. Tissue Array Preparation. We followed the methods described by Wang et al. [18].

2.3. IHC Analysis. We followed the methods of Wang et al. [18]. The primary antibodies consisted of anti-resistin mouse monoclonal antibody (clone C-10, diluted at 1:25; Santa Cruz Biotechnology, Santa Cruz, USA), anti-EGFR rabbit polyclonal antibody (clone 1005, diluted at 1:100; Santa Cruz Biotechnology), anti-ERK1/2 rabbit monoclonal antibody (clone EPR18444, diluted at 1:1000; Abcam, Cambridge, England), ready-to-use anti-ER rabbit monoclonal antibody (clone SP1, Dako), ready-to-use anti-PR mouse monoclonal antibody (clone PgR636, Dako), HercepTest (Dako), and ready-to-use anti-Ki-67 mouse monoclonal antibody (clone MIB-1, Dako).
2.4. Assessment of Staining. The entire section was scanned and scored independently by 2 pathologists. Resistin and EGFR expression staining intensity was scored on a 4-point scale from 0 (negative) to 1 (weak), 2 (moderate), or 3 (strong) [18]. A case was recorded as resistin-positive if the cytoplasmic staining intensity of positive invasive cancer cells was 2 or 3; 3 was deemed strongly positive [8]. Cases were EGFR-positive when $\geq 10 \%$ of the invasive tumor cells had a membranous staining intensity of 1,2 , or $3 ; 2$ and 3 were considered to be strongly positive [11]. ER or PR positivity was satisfied if the percentage of positive invasive cancer cells (nuclear staining) was $\geq 1 \%$ [19]. HER2 status was determined by the 2018 American Society of Clinical Oncology/College of American Pathologists guidelines for HER2 testing in breast cancer [20]. High levels of resistin and EGFR expression were expressed as positive or strongly positive for resistin and strongly positive for EGFR.

2.5. Patient Follow-Up. We followed the methods of Wang et al. [18].

2.6. Statistical Analysis. We followed the methods of Wang et al. [18]. Multivariate analysis using the Cox proportional hazard model was performed to investigate independent factors prognostic of RFS and OS.

\section{Results}

3.1. Expression of Resistin in Breast Tissue and Its Relationship with Clinicopathological Variables. The rate of positive resistin expression in breast cancer tissue specimens was $83.2 \%$ (326/392), which included 82 (20.9\%) strongly positive cases; corresponding rates in normal breast tissue specimens were $23.8 \%(10 / 42)$ and $0.0 \%$ (0/42), respectively. Rates of positive and strongly positive resistin expression were significantly higher in breast cancer tissue than in normal breast tissue $(P<0.001$ and $P=0.001$, respectively) (Table 1). As shown in Table 2, we observed significant associations in breast cancer tissue specimens between positive resistin expression and several clinical parameters, including tumor size $(P=0.012)$, grade $(P<0.001)$, stage $(P=0.042)$, ER $(P<0.001)$ and PR $(P<0.001)$ status, HER2 status $(P=0.021)$, and molecular classification $(P<0.001)$. Strongly positive resistin expression was associated with higher tumor grade $(P<0.001)$, ER $(P<0.001)$ and $\mathrm{PR}$ $(P<0.001)$ status, HER2 status $(P=0.001)$, and molecular classification $(P<0.001)$.

3.2. Correlation of Resistin and EGFR Expression in Breast Cancer. Rates of positive and strongly positive EGFR expression in breast cancer tissues were 52.0\% (204/392) and 32.4\% (127/392), respectively. When we analyzed the relationship between resistin and EGFR expression in breast cancer tissue specimens, we found significantly higher levels of positive or strongly positive EGFR expression among resistin-positive cases (57.7\% [188/326] and 35.9\% [117/326], respectively) compared with resistin-negative cases $(24.2 \%$ [16/66] $(P<0.001)$ and $15.2 \%$ [10/66] $(P=0.001)$, respectively, Table 3). Spearman correlation analysis revealed significantly positive correlations between positive levels of resistin expression and EGFR- 
TABLE 1: Resistin expression in breast tissue specimens obtained from 392 Chinese Han patients.

\begin{tabular}{lcccrr}
\hline Tissue & No. & Negative & Positive & \multicolumn{2}{c}{ Resistin expression } \\
& & $n(\%)$ & $n(\%)$ & Not strongly positive & \multicolumn{2}{c}{$\begin{array}{c}\text { Strongly positive } \\
n(\%)\end{array}$} & $0(0.0 \%)$ \\
\hline Normal & 42 & $32(76.2 \%)$ & $10(23.8 \%)$ & $42(100.0 \%)$ & $310(79.1 \%)$ \\
Tumor & 392 & $66(16.8 \%)$ & $326(83.2 \%)^{*}$ & $32(20.9 \%)^{* *}$ \\
\hline
\end{tabular}

${ }^{*} P<0.001 ;{ }^{* *} P=0.001$.

TABle 2: Association of resistin expression with clinicopathological parameters in 392 Chinese Han patients with breast cancer.

\begin{tabular}{|c|c|c|c|c|c|}
\hline Parameters & No. of patients & $\begin{array}{l}\text { Positive resistin expression } \\
n(\%)\end{array}$ & $P$ value & $\begin{array}{l}\text { Strongly positive resistin expression } \\
n(\%)\end{array}$ & $P$ value \\
\hline \multicolumn{6}{|l|}{ Age (years) } \\
\hline$\leq 35$ & 19 & $16(84.2 \%)$ & \multirow[t]{3}{*}{0.966} & $6(31.6 \%)$ & \multirow[t]{3}{*}{0.466} \\
\hline $35-55$ & 238 & $197(82.8 \%)$ & & $47(19.7 \%)$ & \\
\hline$>55$ & 135 & $113(83.7 \%)$ & & $29(21.5 \%)$ & \\
\hline \multicolumn{6}{|l|}{ Tumor size $(\mathrm{cm})$} \\
\hline$\leq 2$ & 181 & $140(77.3 \%)$ & \multirow[t]{3}{*}{0.012} & $33(18.2 \%)$ & \multirow[t]{3}{*}{0.466} \\
\hline $2-5$ & 192 & $168(87.5 \%)$ & & $45(23.4 \%)$ & \\
\hline$>5$ & 19 & $18(94.7 \%)$ & & $4(21.1 \%)$ & \\
\hline \multicolumn{6}{|c|}{ Lymph node metastases } \\
\hline No & 199 & $159(79.9 \%)$ & \multirow[t]{2}{*}{0.080} & $45(22.6 \%)$ & \multirow[t]{2}{*}{0.402} \\
\hline Yes & 193 & $167(86.5 \%)$ & & $37(19.2 \%)$ & \\
\hline \multicolumn{6}{|l|}{ Tumor grade } \\
\hline I & 19 & $11(57.9 \%)$ & \multirow[t]{3}{*}{$<0.001$} & $0(0.0 \%)$ & \multirow[t]{3}{*}{$<0.001$} \\
\hline II & 265 & $215(81.1 \%)$ & & $47(17.7 \%)$ & \\
\hline III & 108 & $100(92.6 \%)$ & & $35(32.4 \%)$ & \\
\hline \multicolumn{6}{|l|}{ Tumor stage } \\
\hline I & 106 & $80(75.5 \%)$ & \multirow[t]{4}{*}{0.042} & $20(18.9 \%)$ & \multirow[t]{4}{*}{0.656} \\
\hline II & 188 & $163(86.7 \%)$ & & $43(22.9 \%)$ & \\
\hline III & 98 & $83(84.7 \%)$ & & $19(19.4 \%)$ & \\
\hline IV & 0 & $0(0.0 \%)$ & & $0(0.0 \%)$ & \\
\hline \multicolumn{6}{|l|}{ Estrogen receptor } \\
\hline Negative & 148 & $139(93.9 \%)$ & \multirow[t]{2}{*}{$<0.001$} & $59(39.9 \%)$ & \multirow[t]{2}{*}{$<0.001$} \\
\hline Positive & 244 & $187(76.6 \%)$ & & $23(9.4 \%)$ & \\
\hline \multicolumn{6}{|c|}{ Progesterone receptor } \\
\hline Negative & 192 & $177(92.2 \%)$ & \multirow[t]{2}{*}{$<0.001$} & $63(32.8 \%)$ & \multirow[t]{2}{*}{$<0.001$} \\
\hline Positive & 200 & $149(74.5 \%)$ & & $19(9.5 \%)$ & \\
\hline \multicolumn{6}{|l|}{ HER2 expression } \\
\hline Negative $\left(0-1^{+}\right)$ & 184 & $147(79.9 \%)$ & \multirow[t]{3}{*}{0.021} & $34(18.5 \%)$ & \multirow[t]{3}{*}{0.001} \\
\hline Equivocal $\left(2^{+}\right)$ & 107 & $86(80.4 \%)$ & & $14(13.1 \%)$ & \\
\hline Positive $\left(3^{+}\right)$ & 101 & $93(92.1 \%)$ & & $34(33.7 \%)$ & \\
\hline \multicolumn{6}{|c|}{ Molecular classification } \\
\hline Luminal A & 147 & $104(70.7 \%)$ & \multirow[t]{4}{*}{$<0.001$} & $12(8.2 \%)$ & \multirow[t]{4}{*}{$<0.001$} \\
\hline Luminal B & 101 & $87(86.1 \%)$ & & $11(10.9 \%)$ & \\
\hline HER2-enriched & 71 & $66(93.0 \%)$ & & $33(46.5 \%)$ & \\
\hline TNBC & 73 & $69(94.5 \%)$ & & $26(35.6 \%)$ & \\
\hline
\end{tabular}

Abbreviations: HER2 = human epidermal growth factor receptor 2; TNBC = triple-negative breast cancer.

positive or strongly positive expression in breast cancer tissue specimens $(r=0.250$ and $P<0.001$ and $r=0.166$ and $P=$ 0.001 , respectively). Similarly, as shown in Table 4 and
Figure 1, significantly higher levels of positive or strongly positive EGFR expression were identified in cases that were strongly positive for resistin $(82.9 \%$ [68/82] and 51.2\% [42/82], 
TABLE 3: Relationships between positive resistin expression and EGFR expression in 392 Chinese Han patients with breast cancer.

\begin{tabular}{|c|c|c|c|c|c|}
\hline \multirow[b]{2}{*}{ Patients } & \multirow[b]{2}{*}{ No. } & \multicolumn{4}{|c|}{ EGFR expression } \\
\hline & & $\begin{array}{l}\text { Negative } \\
n(\%)\end{array}$ & $\begin{array}{l}\text { Positive } \\
n(\%)\end{array}$ & $\begin{array}{c}\text { Not strongly positive } \\
n(\%)\end{array}$ & $\begin{array}{c}\text { Strongly positive } \\
n(\%)\end{array}$ \\
\hline Resistin negative & 66 & $50(75.8 \%)$ & $16(24.2 \%)$ & $56(84.8 \%)$ & $10(15.2 \%)$ \\
\hline Resistin positive & 326 & $138(42.3 \%)$ & $188(57.7 \%)^{*}$ & $209(64.1 \%)$ & $117(35.9 \%)^{* *}$ \\
\hline
\end{tabular}

${ }^{*} P<0.001 ;{ }^{* *} P=0.001$.

TABLE 4: Relationships between strongly positive resistin expression and EGFR expression in 392 Chinese Han patients with breast cancer.

\begin{tabular}{|c|c|c|c|c|c|}
\hline \multirow[b]{2}{*}{ Patients } & \multirow[b]{2}{*}{ No. } & \multicolumn{4}{|c|}{ EGFR expression } \\
\hline & & $\begin{array}{c}\text { Negative } \\
n(\%)\end{array}$ & $\begin{array}{c}\text { Positive } \\
n(\%)\end{array}$ & $\begin{array}{c}\text { Not strongly positive } \\
n(\%)\end{array}$ & $\begin{array}{c}\text { Strongly positive } \\
n(\%)\end{array}$ \\
\hline Not strongly positive for resistin & 310 & $174(56.1 \%)$ & $136(43.9 \%)$ & $225(72.6 \%)$ & $85(27.4 \%)$ \\
\hline Strongly positive for resistin & 82 & $14(17.1 \%)$ & $68(82.9 \%)^{*}$ & $40(48.8 \%)$ & $42(51.2 \%)^{* *}$ \\
\hline
\end{tabular}

${ }^{*} P<0.001 ;{ }^{* *} P<0.001$.

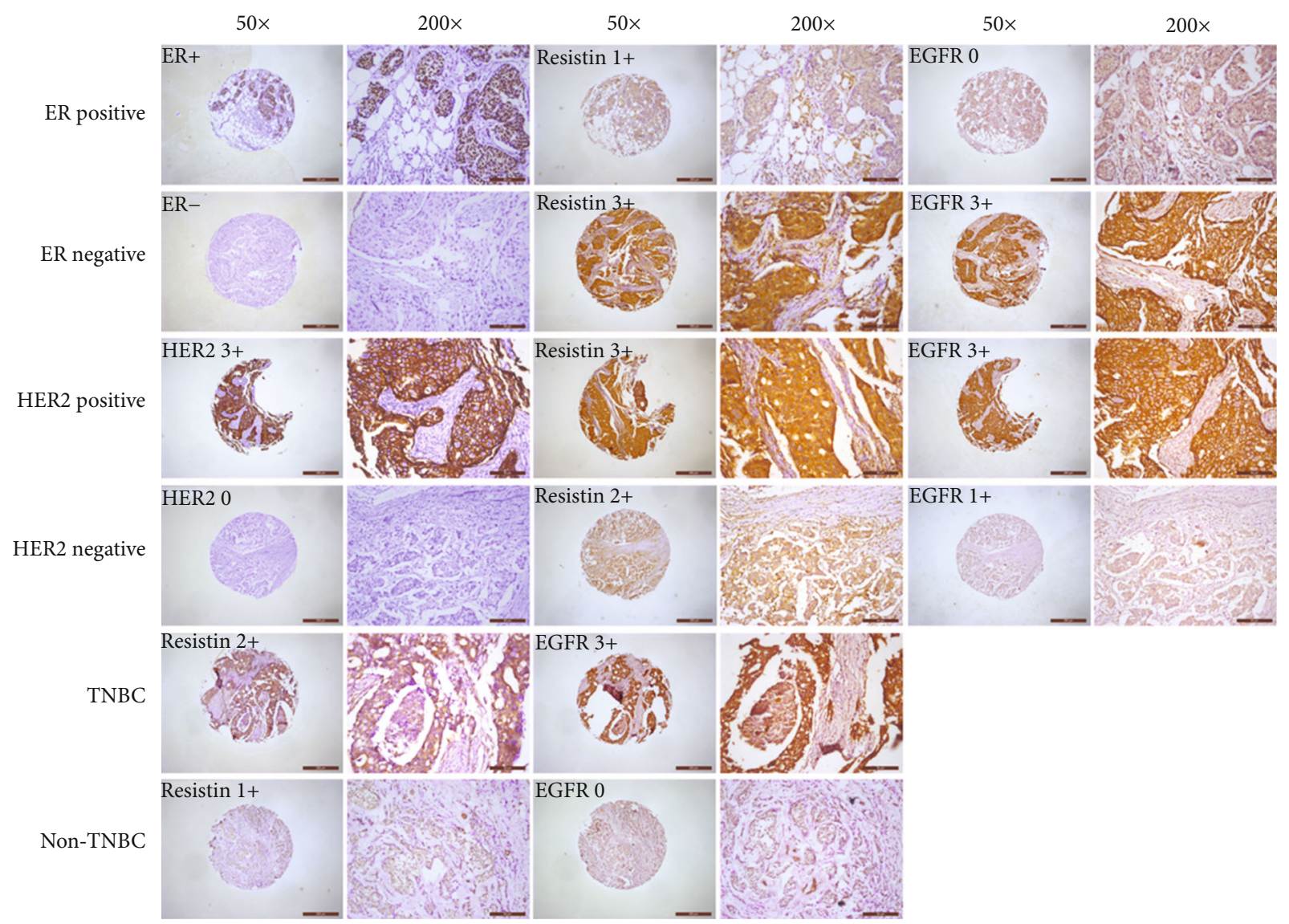

FIGURE 1: Resistin and EGFR protein expression was analyzed in ER-positive/ER-negative, HER-positive/HER-negative, TNBC/non-TNBC human breast cancer tissues by immunohistochemical staining. Cancer tissue microarrays were immune-stained with anti-resistin, antiEGFR, anti-ER, and anti-HER2 antibodies. Representative images of stained tissues are shown.

respectively) compared with those that were not strongly resistin-positive $(43.9 \%$ [136/310] $(P<0.001)$ and $27.4 \%$ [85/310] $(P<0.001)$, respectively). Spearman correlation analysis revealed significantly positive correlations between strongly positive levels of resistin expression and EGFR-positive or strongly positive expression in breast cancer tissue specimens ( $r=0.318$ and $P<0.001$ and $r=0.207$ and $P<0.001$, respectively). Similarly, a positive correlation was observed between staining intensity scores of resistin and EGFR in breast cancer tissues $(r 2=0.1124, P<0.001)$ (Figure 2). 


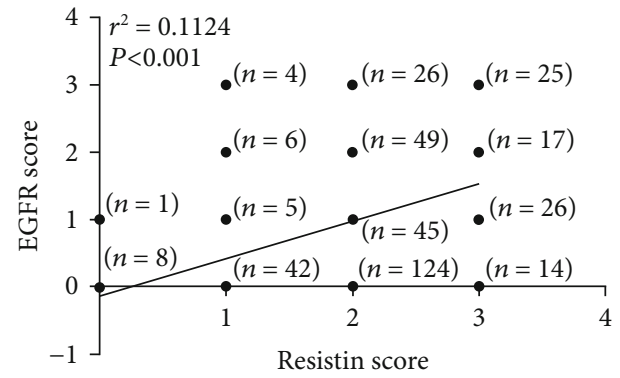

FIgURE 2: A positive correlation was observed between levels of resistin and EGFR expression in breast cancer tissues.

\subsection{Combined High Resistin and EGFR Expression Is} Associated with Worse Survival in Breast Cancer. RFS and OS were worse in patients with resistin-positive tumors compared with those who were resistin-negative, and RFS was worse in patients with strongly resistin-positive tumors compared with those whose tumors were not strongly resistinpositive, while patients whose tumors were strongly resistin-positive experienced worse RFS compared with patients whose tumors were weakly resistin-positive and those with resistin-negative tumors $(P=0.237, P=0.128, P$ $=0.171$, and $P=0.105$, respectively) (Figures 3(a)-3(f)). Similarly, RFS was worse for EGFR-positive patients compared with EGFR-negative patients, while RFS and OS were worse for strongly EGFR-positive patients compared with those without strongly EGFR-positive tumors $(P=0.327, P$ $=0.055$, and $P=0.292$, respectively) (Figures $3(\mathrm{~g})-3(\mathrm{j})$ ).

As shown in Figures $3(\mathrm{k})-3(\mathrm{n})$, patients whose primary tumors were both resistin-positive and strongly EGFRpositive $(n=74)$ had a mean RFS of 51.5 months (an estimated 5-year RFS rate of 68.9\%); patients whose tumors were either resistin-positive or strongly EGFR-positive $(n=129)$ had a mean RFS of 54.3 months (an estimated 5-year RFS rate of $83.7 \%$ ), while patients whose tumors were resistinnegative and not strongly EGFR-positive $(n=36)$ had a mean RFS of 54.1 months (an estimated 5-year RFS rate of $83.3 \%$, $P=0.037)$. Patients whose tumors were both resistinpositive and strongly EGFR-positive experienced worse OS compared with patients whose tumors were either resistinpositive or strongly EGFR-positive and patients whose tumors were resistin-negative and not strongly EGFRpositive $(P=0.099)$.

As shown in Figures 3(o)-3(r), patients whose primary tumors were both strongly resistin-positive and strongly EGFR-positive $(n=29)$ had a mean RFS of 49.1 months (an estimated 5-year RFS rate of 62.1\%); patients whose tumors were either strongly resistin-positive or strongly EGFR-positive $(n=73)$ had a mean RFS of 54.2 months (an estimated 5-year RFS rate of 79.5\%), and patients whose tumors were not strongly resistin-positive and not strongly EGFR-positive $(n=137)$ had a mean RFS of 53.9 months (an estimated 5-year RFS rate of $82.5 \%, P=0.035$ ). Patients whose tumors were both strongly resistin-positive and strongly EGFR-positive experienced worse OS compared with patients whose tumors were either strongly resistinpositive or strongly EGFR-positive and patients whose tumors were not strongly positive for either protein $(P=0.453)$. Cox proportional hazards regression analysis did not reveal any significant associations between resistin-positive and strongly EGFR-positive tumor tissue (hazard ratio $(\mathrm{HR})=0.736,95 \% \mathrm{CI}=0.198-2.734, \quad P=$ $0.648 ; 0.765,0.110-5.323, P=0.786)$, strongly resistinpositive and strongly EGFR-positive tumor tissue (0.784, $0.273-2.255, \quad P=0.652 ; \quad 0.359, \quad 0.100-1.294, \quad P=0.118)$, and RFS or OS.

3.4. Combined High Resistin and EGFR Expression Is Associated with Survival in Non-TNBC or TNBC, ERNegative or ER-Positive, HER-Negative or HER2-Positive Breast Cancer. We analyzed the effect of combined high expression of resistin and EGFR on the prognosis of non-TNBC or TNBC, ER-negative or ER-positive, HERnegative or HER2-positive tumors. As shown in Figures 4(a) and 4(b), non-TNBC patients whose primary tumors were both resistin-positive and strongly EGFRpositive $(n=61)$ had a mean RFS of 51.3 months (an estimated 5-year RFS rate of $67.2 \%$ ); patients whose tumors were either resistin-positive or strongly EGFR-positive $(n=111)$ had a mean RFS of 55.2 months (an estimated 5 -year RFS rate of $86.5 \%$ ), while patients whose tumors were resistin-negative and not strongly EGFR-positive $(n=36)$ had a mean RFS of 54.1 months (an estimated 5 -year RFS rate of $83.3 \%, P=0.024)$. Non-TNBC patients whose tumors were both resistin-positive and strongly EGFR-positive experienced worse OS compared with patients whose tumors were either resistin-positive or strongly EGFR-positive and also patients whose tumors were resistin-negative and not strongly EGFR-positive $(P=0.065)$. As shown in Figures 4(c) and 4(d), nonTNBC patients whose primary tumors were both strongly resistin-positive and strongly EGFR-positive $(n=23)$ had a mean RFS of 48.3 months (an estimated 5-year RFS rate of $60.9 \%$ ); patients whose tumors were either strongly resistin-positive or strongly EGFR-positive $(n=59)$ had a mean RFS of 54.5 months (an estimated 5-year RFS rate of $78.0 \%$ ), and patients whose tumors were not strongly resistin-positive and not strongly EGFR-positive $(n=126)$ had a mean RFS of 54.6 months (an estimated 5-year RFS rate of $84.9 \%, P=0.010)$. Non-TNBC patients whose tumors were both strongly resistin-positive and strongly EGFR-positive experienced worse OS compared with patients whose tumors were either strongly resistinpositive or strongly EGFR-positive and patients whose tumors were not strongly positive for either protein $(P=0.135)$.

As shown in Figures 4(e)-4(h), in TNBC, the prognosis of tumors that were both resistin-positive and strongly EGFR-positive, or strongly resistin-positive and strongly EGFR-positive, did not differ significantly from that of other breast cancer groups. Similarly, in ER-negative or ERpositive and HER2-negative or HER2-positive disease, the prognosis of tumors that were both resistin-positive and strongly EGFR-positive, or strongly resistin-positive and strongly EGFR-positive, did not differ significantly from that of other groups. 


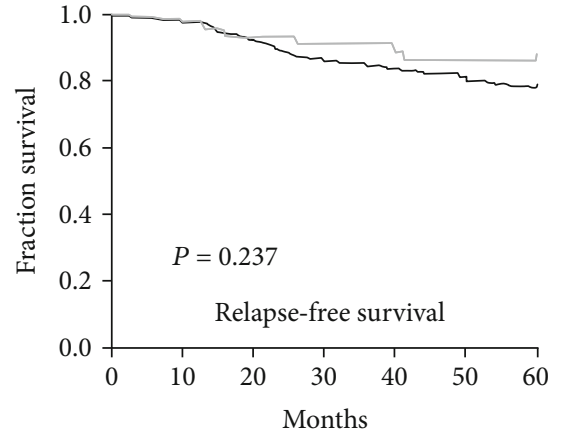

Resistin-negative: $n=43$

$\perp$ Resistin-positive: $n=196$

(a)

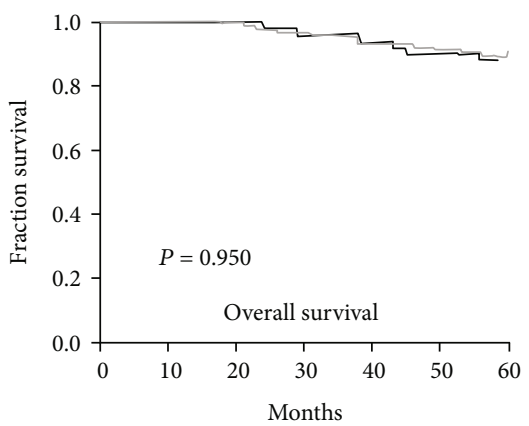

$\ldots$ Not strongly resistin-positive: $n=189$ $\_$Strongly resistin-positive: $n=50$

(d)

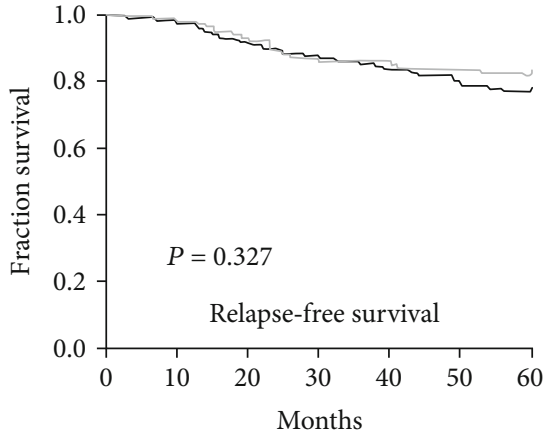

EGFR-negative: $n=116$

- EGFR-positive: $n=123$

(g)

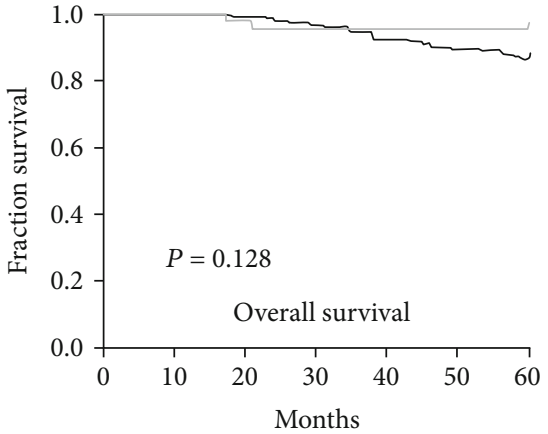

Resistin-negative: $n=43$

_ Resistin-positive: $n=196$

(b)

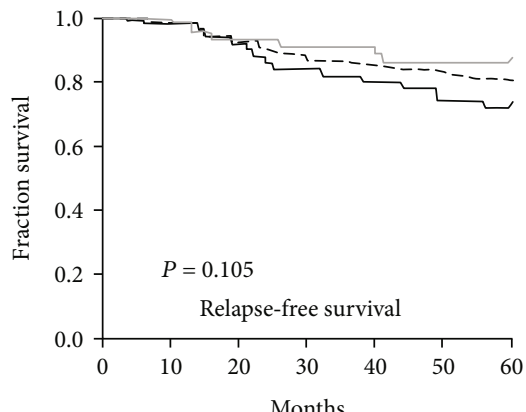

1 Resistin-negative $(0-1+): n=43$

- $\perp$ - Weakly resistin-positive $(2+): n=146$

$\_$Strongly resistin-positive (3+): $n=50$

(e)

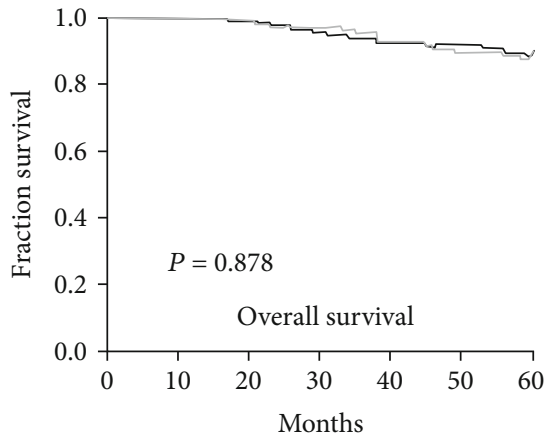

EGFR-negative: $n=116$

ـ EGFR-positive: $n=123$

(h)

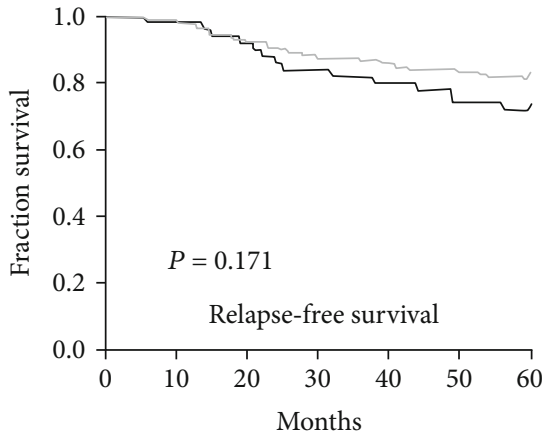

Not strongly resistin-positive: $n=189$ ـ Strongly resistin-positive: $n=50$

(c)

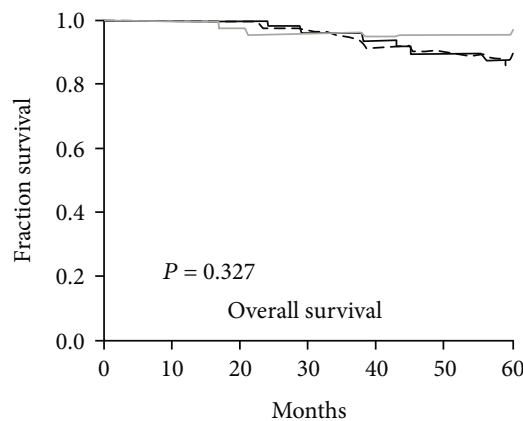

1 Resistin-negative $(0-1+): n=43$

- $\perp$ - Weakly resistin-positive $(2+): n=146$

$\perp$ Strongly resistin-positive (3+): $n=50$

(f)

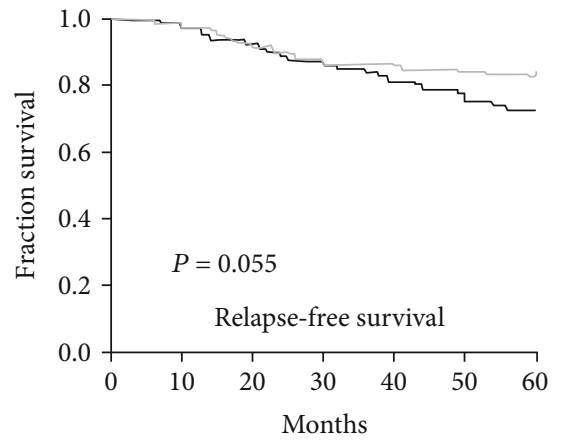

- Not strongly EGFR-positive: $n=158$ — Strongly EGFR-positive: $n=81$

(i)

Figure 3: Continued. 


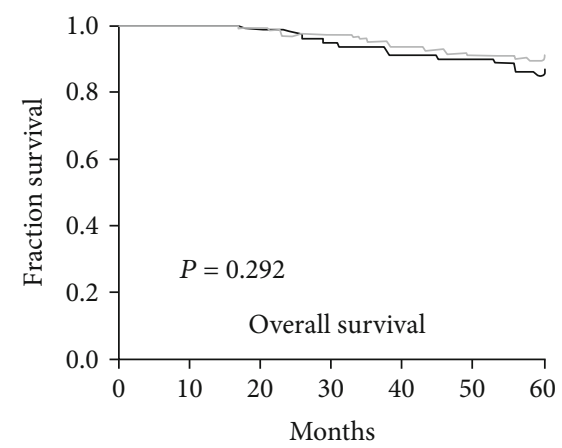

Not strongly EGFR-positive: $n=158$ - Strongly EGFR-positive: $n=81$

(j)

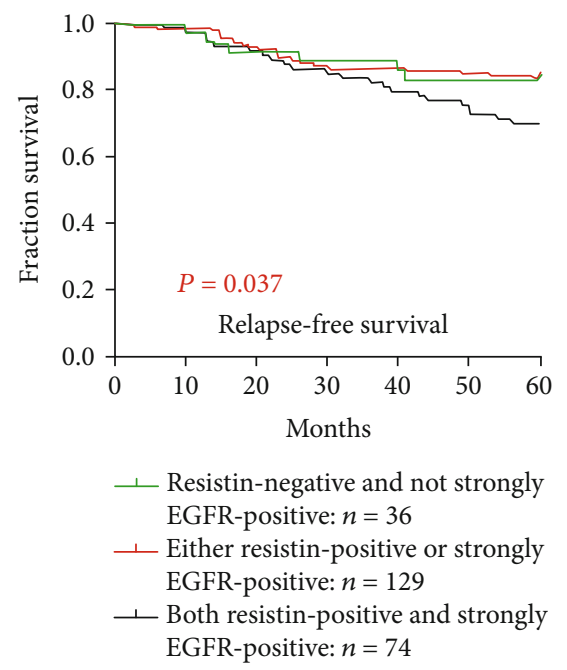

(m)

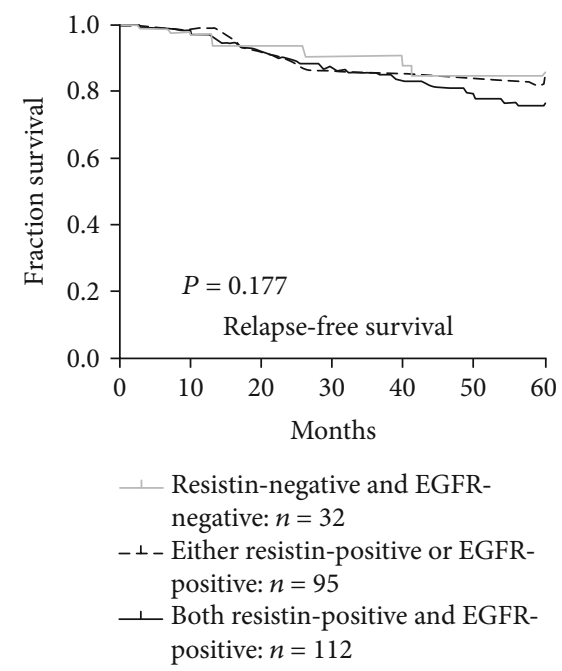

(k)

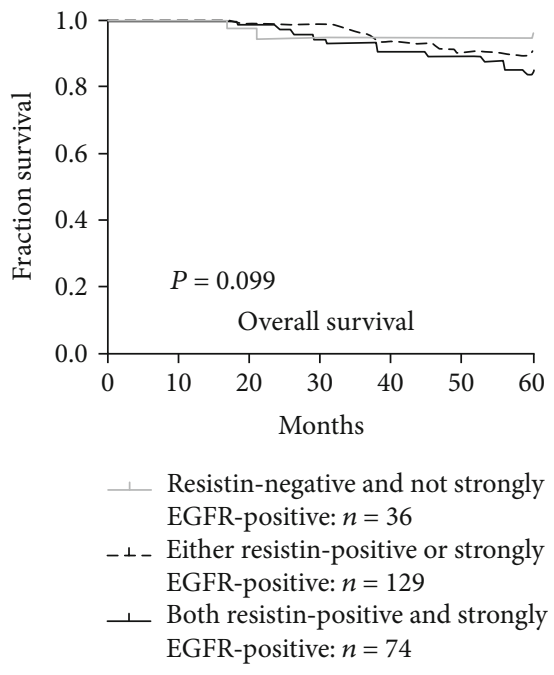

(n)

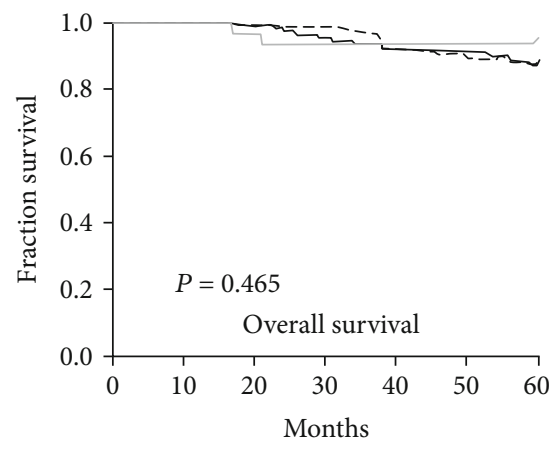

Resistin-negative and EGFRnegative: $n=32$

- - E Either resistin-positive or EGFRpositive: $n=95$

$\perp$ Both resistin-positive and EGFRpositive: $n=112$

(1)

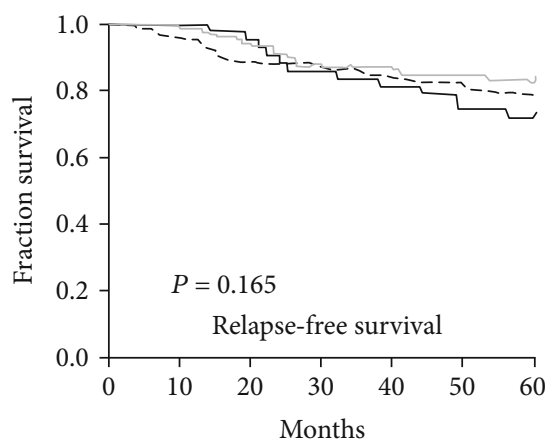

$\ldots$ Not strongly resistin-positive and EGFR-negative: $n=109$

$\_\_$- Either strongly resistin-positive or EGFR-positive: $n=87$

1 Both strongly resistin-positive and EGFR-positive: $n=43$

(o)

Figure 3: Continued. 


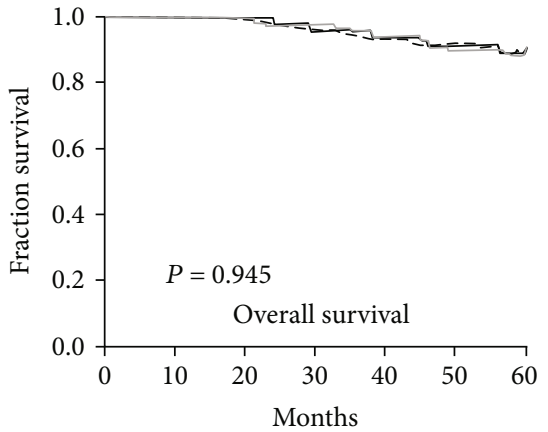

Not strongly resistin-positive and EGFR-negative: $n=109$

- $\perp$ - Either strongly resistin-positive or EGFR-positive: $n=87$

$\perp$ Both strongly resistin-positive and EGFR-positive: $n=43$

(p)

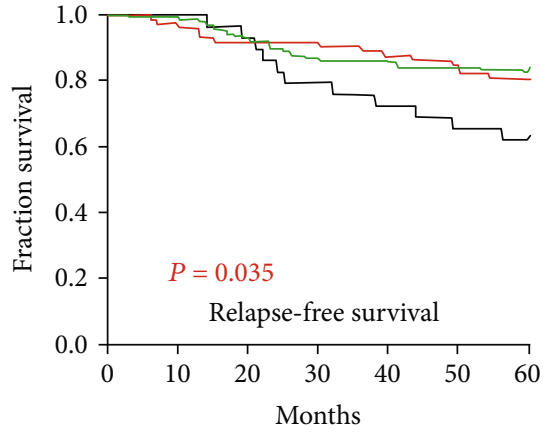

- Not strongly resistin-positive and not strongly EGFR-positive: $n=137$

+ Either strongly resistin-positive or strongly EGFR-positive: $n=73$

1 Both strongly resistin-positive and strongly EGFR-positive: $n=29$

(q)

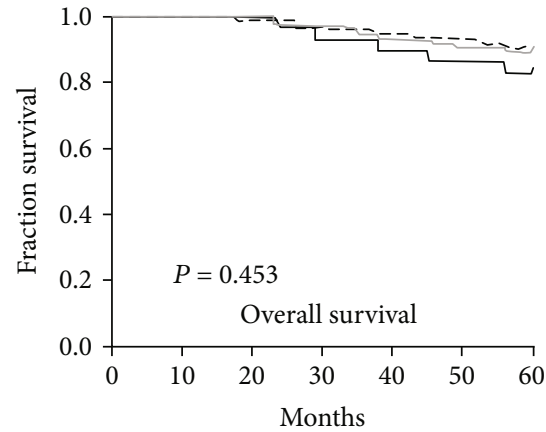

1 Not strongly resistin-positive and not strongly EGFR-positive: $n=137$

- - Either strongly resistin-positive or strongly EGFR-positive: $n=73$

$\ldots$ Both strongly resistin-positive and strongly EGFR-positive: $n=29$

(r)

FIGURE 3: Levels of resistin and EGFR expression were associated with survival of patients with breast cancer. Associations were analyzed between resistin-positive expression and (a) relapse-free survival (RFS) and also (b) overall survival (OS). Associations were analyzed between strongly positive resistin expression and (c) RFS and also (d) OS. Associations were analyzed between strongly positive and weakly-positive resistin expression and (e) RFS and also (f) OS. Associations were analyzed between EGFR-positive expression and (g) RFS and also (h) OS. Associations were analyzed between strongly positive EGFR expression and (i) RFS and also (j) OS. Kaplan-Meier curves for (k) RFS and (l) OS in breast cancer patients with combined resistin-positive and EGFR-positive expression. Kaplan-Meier curves for (m) RFS and (n) OS in breast cancer patients with combined resistin-positive and strongly positive EGFR expression. KaplanMeier curves for (o) RFS and (p) OS in breast cancer patients with combined strongly positive resistin and EGFR-positive expression. Kaplan-Meier curves for (q) RFS and (r) OS in breast cancer patients with combined strongly positive resistin and strongly positive EGFR expression. $P$ values were calculated using the Mantel-Cox log-rank test.

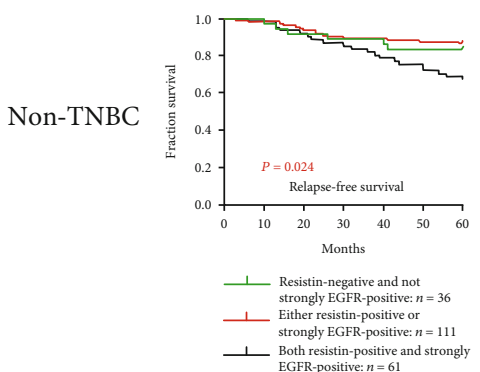

(a)

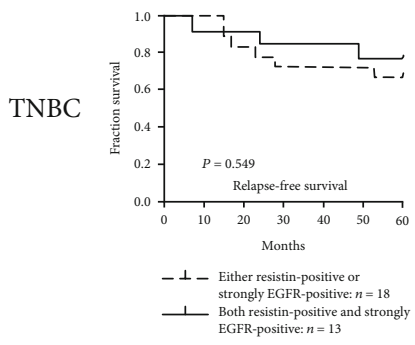

(e)

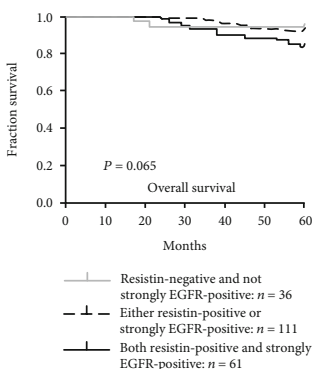

(b)

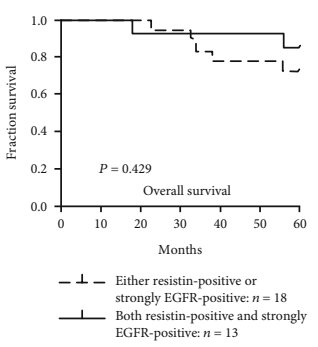

(f)

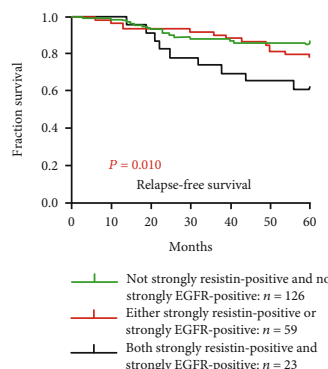

(c)

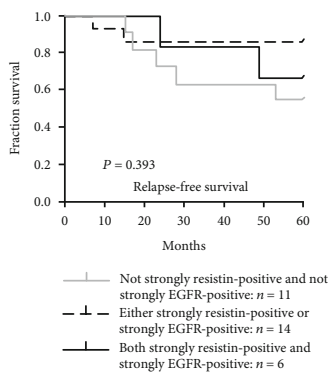

(g)

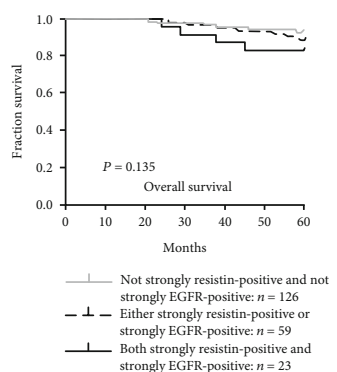

(d)

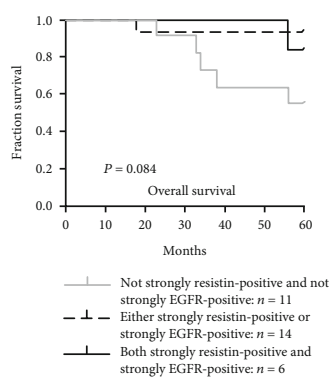

(h)

FIGURE 4: Combined high levels of resistin and EGFR are associated with survival in non-TNBC and TNBC disease. Kaplan-Meier curves for (a) RFS and (b) OS in non-TNBC patients with combined resistin-positive and strongly positive EGFR expression. Kaplan-Meier curves for (c) RFS and (d) OS in non-TNBC patients with combined strongly positive resistin and strongly positive EGFR expression. Kaplan-Meier curves for (e) RFS and (f) OS in TNBC patients with combined resistin-positive and strongly positive EGFR expression. Kaplan-Meier curves for (g) RFS and (h) OS in TNBC patients with combined strongly positive resistin and strongly positive EGFR expression. $P$ values were calculated using the Mantel-Cox log-rank test. 


\section{Discussion}

The cytokine resistin participates in several physiological and pathological processes, including metabolism, inflammation, autoimmunity, and various cancers, including breast cancer [1-6, 21-24]. Higher levels of circulating resistin have been linked to a risk of developing breast cancer $[1,2]$, and significantly elevated resistin expression has been documented in patients with breast cancer [3-7]. Our previous study found upregulated resistin expression in breast cancer tissue than in normal breast tissue [8], but in that study, we did not analyze the relationship between the high expression of resistin and the clinicopathological characteristics of breast cancer patients. In this study, we observed that both positive and strongly positive rates of resistin expression are significantly higher in breast cancer tissue specimens compared with normal breast tissue and that both positive and strongly positive resistin expressions are significantly associated with a number of clinicopathological parameters in breast cancer patients. For example, positive resistin expression was correlated with larger tumor size, higher tumor grade, higher clinical stage, and HER2-positive expression; strongly positive resistin expression was correlated with higher tumor grade and HER2-positive expression. Since higher tumor grade and HER2-positive expression are known to be more aggressive and to have a poor prognosis $[15,25]$, this finding indicates that the high expression of resistin may be closely related to highly invasive breast cancer and a poor prognosis. Our results also revealed significantly increased levels of resistin expression in ER- and PR-negative disease, and evidence has shown that resistin promotes invasion and migration of the TNBC cell line MDA-MB-231 [26]. Since hormone receptor-negative breast cancer patients are unsuitable candidates for endocrine therapy, further research should examine whether these patients may benefit from the downregulation of resistin. We also found in this study that resistin is highly expressed in TNBC and HER2-enriched subtypes. TNBC is associated with a more aggressive clinical course and poorer prognosis than other types of breast cancer [2730]. Our findings suggest that resistin may be a potential marker for the treatment of patients with TNBC.

Our previous study have shown that EGFR promotes breast cancer invasion through downstream p44/p42 MAPK (ERK1/2) signaling [11], and resistin is known to enhance angiogenesis in human osteosarcoma cells via the ERK1/2 signaling [12]. In this study, our data revealed a significantly positive correlation between positive levels of resistin and ERK1/2 in breast cancer tissues (Supplementary Materials, Tables S1 and S2). Another study has shown that resistin activates Toll-like receptor 4 (TLR4) and engages with the Src pathway which activated the EGFR phosphorylation and increased epithelial-mesenchymal transition (EMT), subsequently inducing the migration and invasion of lung adenocarcinoma [13]. In addition, resistin facilitates breast cancer progression via TLR4/nuclear factor kappa-light-chainenhancer of activated B cells (NF- $\kappa \mathrm{B}) /$ signal transducer and activator of transcription 3 (STAT3) signaling pathway- mediated induction of mesenchymal phenotypes and stemness properties [31]. These results suggest that both resistin and EGFR high expression tumor has more potent tumor metastasis and recurrence, and there may be a relationship between resistin and EGFR expression in breast cancer. In this study, we found that tumors that are resistinpositive or strongly resistin-positive are significantly positively correlated with EGFR-positive and strongly EGFR-positive expression. We therefore sought to determine the clinical prognostic significance of our observed highly significant correlation between resistin and EGFR expression in breast cancer tissue.

In this study, both resistin and EGFR impacted adversely upon survival in breast cancer, but neither protein alone had a significant impact. Breast cancer patients whose tumors were both resistin-positive and strongly EGFR-positive, or were both strongly resistin-positive and strongly EGFR-positive, had worse RFS than all other breast cancer patients. In the non-TNBC and TNBC subgroup analyses, similar results were observed with non-TNBC, but not with TNBC. This may be due to the fact that there were too few cases for analysis. Further study should examine the effects of combined high resistin and EGFR expression on the prognosis of patients with TNBC. These results suggest that resistin and EGFR are potential clinical biomarkers of disease progression and prognosis in breast cancer and that simultaneously targeting these proteins is a potentially useful therapeutic strategy in this disease.

\section{Abbreviations}

EGFR: Epidermal growth factor receptor

IHC: Immunohistochemistry

MAPK: Mitogen-activated protein kinase

ERK1/2: Extracellular signal-regulated kinase 1/2

RFS: $\quad$ Relapse-free survival

OS: Overall survival.

\section{Data Availability}

All data generated or analyzed during this study are included in this published article.

\section{Conflicts of Interest}

The authors declare no conflicts of interest.

\section{Authors' Contributions}

YZ, YW, HJL, QW, and LLJ performed the experiments. CQW and BFH analyzed the data. CQW and LST designed the experiments. JKS was responsible for the management of the clinical samples. CQW and YZ wrote the manuscript. CHT and LST revised the manuscript. All authors have read and approved the final manuscript. Yue Zeng and Chih-Hsin Tang contributed equally to this manuscript. 


\section{Acknowledgments}

This work was supported by a grant from the National Natural Science Foundation of China (No. 81802660).

\section{Supplementary Materials}

Relationships between resistin expression and ERK1/2 expression in patients with breast cancer (Supplementary Materials)

\section{References}

[1] G. S. Christodoulatos, N. Spyrou, J. Kadillari, S. Psallida, and M. Dalamaga, "The role of adipokines in breast cancer: current evidence and perspectives," Current Obesity Reports, vol. 8, no. 4, pp. 413-433, 2019.

[2] Y. Gui, Q. Pan, X. Chen, S. Xu, X. Luo, and L. Chen, “The association between obesity related adipokines and risk of breast cancer: a meta-analysis," Oncotarget, vol. 8, no. 43, pp. 75389-75399, 2017.

[3] J. H. Kang, B. Y. Yu, and D. S. Youn, "Relationship of serum adiponectin and resistin levels with breast cancer risk," Journal of Korean Medical Science, vol. 22, no. 1, pp. 117-121, 2007.

[4] W. K. Hou, Y. X. Xu, T. Yu et al., "Adipocytokines and breast cancer risk," Chinese Medical Journal, vol. 120, no. 18, pp. 1592-1596, 2007.

[5] C. A. Sun, M. H. Wu, C. H. Chu et al., "Adipocytokine resistin and breast cancer risk," Breast Cancer Research and Treatment, vol. 123, no. 3, pp. 869-876, 2010.

[6] A. M. Assiri and H. F. Kamel, "Evaluation of diagnostic and predictive value of serum adipokines: leptin, resistin and visfatin in postmenopausal breast cancer," Obesity Research \& Clinical Practice, vol. 10, no. 4, pp. 442-453, 2016.

[7] Y. C. Lee, Y. J. Chen, C. C. Wu, S. Lo, M. F. Hou, and S. S. Yuan, "Resistin expression in breast cancer tissue as a marker of prognosis and hormone therapy stratification," Gynecologic Oncology, vol. 125, no. 3, pp. 742-750, 2012.

[8] C. Q. Wang, C. H. Tang, H. E. Tzeng et al., "Impacts of RETN genetic polymorphism on breast cancer development," Journal of Cancer, vol. 11, no. 10, pp. 2769-2777, 2020.

[9] N. E. Hynes and H. A. Lane, "ERBB receptors and cancer: the complexity of targeted inhibitors," Nature Reviews Cancer, vol. 5, no. 5, pp. 341-354, 2005.

[10] R. N. Jorissen, F. Walker, N. Pouliot, T. P. Garrett, C. W. Ward, and A. W. Burgess, "Epidermal growth factor receptor: mechanisms of activation and signalling," Experimental Cell Research, vol. 284, no. 1, pp. 31-53, 2003.

[11] C. Q. Wang, Y. Li, B. F. Huang et al., "EGFR conjunct FSCN1 as a novel therapeutic strategy in triple-negative breast cancer," Scientific Reports, vol. 7, no. 1, article 15654, 2017.

[12] H. C. Tsai, S. P. Cheng, C. K. Han et al., "Resistin enhances angiogenesis in osteosarcoma via the MAPK signaling pathway," Aging, vol. 11, no. 21, pp. 9767-9777, 2019.

[13] W. J. Gong, J. Y. Liu, J. Y. Yin et al., "Resistin facilitates metastasis of lung adenocarcinoma through the TLR4/Src/EGFR/PI3K/NF- $\kappa \mathrm{B}$ pathway," Cancer Science, vol. 109, no. 8, pp. 2391-2400, 2018.

[14] S. R. E. I. Lakhani, S. J. Schnitt, P. H. Tan, and M. J. van de Vijver, WHO Classification of Tumours of the Breast, IARC Press, Lyon, France, 4th Edition edition, 2012.
[15] C. W. Elston and I. O. Ellis, "Pathological prognostic factors in breast cancer. I. The value of histological grade in breast cancer: experience from a large study with long-term follow-up," Histopathology, vol. 19, no. 5, pp. 403-410, 1991.

[16] S. Park, J. S. Koo, M. S. Kim et al., "Characteristics and outcomes according to molecular subtypes of breast cancer as classified by a panel of four biomarkers using immunohistochemistry," The Breast, vol. 21, no. 1, pp. 50-57, 2012.

[17] C. Q. Wang, C. H. Tang, H. T. Chang et al., "Fascin-1 as a novel diagnostic marker of triple-negative breast cancer," Cancer Medicine, vol. 5, no. 8, pp. 1983-1988, 2016.

[18] C. Q. Wang, Y. Wang, B. F. Huang et al., "High expression of both resistin and fascin-1 predicts a poor prognosis in patients with colorectal cancer," BioMed Research International, vol. 2020, Article ID 8753175, 7 pages, 2020.

[19] T. H. Nguyen, V. H. Nguyen, T. L. Nguyen, C. Qiuyin, and T. H. Phung, "Evaluations of biomarker status changes between primary and recurrent tumor tissue samples in breast cancer patients," BioMed Research International, vol. 2019, Article ID 7391237, 7 pages, 2019.

[20] A. C. Wolff, M. E. H. Hammond, K. H. Allison et al., "Human epidermal growth factor receptor 2 testing in breast cancer: American Society of Clinical Oncology/College of American Pathologists clinical practice guideline focused update," Journal of Clinical Oncology, vol. 36, no. 20, pp. 2105-2122, 2018.

[21] M. Filkova, M. Haluzik, S. Gay, and L. Senolt, "The role of resistin as a regulator of inflammation: implications for various human pathologies," Clinical Immunology, vol. 133, no. 2, pp. 157-170, 2009.

[22] M. Zhang, L. Yan, G. J. Wang, and R. Jin, "Resistin effects on pancreatic cancer progression and chemoresistance are mediated through its receptors CAP1 and TLR4," Journal of Cellular Physiology, vol. 234, no. 6, pp. 9457-9466, 2019.

[23] C. C. Zhao, J. Chen, R. F. Niu, Y. Liu, and C. G. Zhang, "Increased resistin suggests poor prognosis and promotes development of lung adenocarcinoma," Oncology Reports, vol. 40, no. 6, pp. 3392-3404, 2018.

[24] W. H. Yang, S. J. Wang, Y. S. Chang, C. M. Su, S. F. Yang, and C. H. Tang, "Association of resistin gene polymorphisms with oral squamous cell carcinoma progression and development," BioMed Research International, vol. 2018, Article ID 9531315, 8 pages, 2018.

[25] M. C. Figueroa-Magalhães, D. Jelovac, R. Connolly, and A. C. Wolff, "Treatment of HER2-positive breast cancer," The Breast, vol. 23, no. 2, pp. 128-136, 2014.

[26] J. O. Lee, N. Kim, H. J. Lee et al., "Resistin, a fat-derived secretory factor, promotes metastasis of MDA-MB-231 human breast cancer cells through ERM activation," Scientific Reports, vol. 6, no. 1, article 18923, 2016.

[27] K. R. Bauer, M. Brown, R. D. Cress, C. A. Parise, and V. Caggiano, "Descriptive analysis of estrogen receptor (ER)negative, progesterone receptor (PR)-negative, and HER2negative invasive breast cancer, the so-called triple-negative phenotype," Cancer, vol. 109, no. 9, pp. 1721-1728, 2007.

[28] P. Boyle, "Triple-negative breast cancer: epidemiological considerations and recommendations," Annals of Oncology, vol. 23, Supplement 6, pp. vi7-vi12, 2012.

[29] S. K. Pal, B. H. Childs, and M. Pegram, "Triple negative breast cancer: unmet medical needs," Breast Cancer Research and Treatment, vol. 125, no. 3, pp. 627-636, 2011. 
[30] C. Y. Li, P. Wang, S. Zhang, Y. Liu, and J. Zhang, "Clinicopathological features and prognosis of triple-negative breast cancer," Zhonghua zhong liu za zhi [Chinese Journal of Oncology], vol. 35, no. 6, pp. 463-467, 2013.

[31] C. H. Wang, P. J. Wang, Y. C. Hsieh et al., "Resistin facilitates breast cancer progression via TLR4-mediated induction of mesenchymal phenotypes and stemness properties," Oncogene, vol. 37, no. 5, pp. 589-600, 2018. 